\title{
How Do Non-Ideal UAV Antennas Affect Air-to-Ground Communications?
}

\author{
M. G. Khoshkholgh*, Keivan Navaie**, Halim Yanikomeroglu ${ }^{\dagger}$, V. C. M. Leung*, and Kang. G. Shin ${ }^{\dagger \dagger}$ \\ * Department of Electrical and Computer Engineering, the University of British Columbia \\ ** School of Computing and Communications, Lancaster University \\ $\dagger$ Department of System and Computer Engineering, Carleton University \\ ${ }^{\dagger \dagger}$ Department of Electrical Engineering and Computer Science, The University of Michigan
}

\begin{abstract}
Analysis of the performance of Unmanned Areal Vehicle (UAV)-enabled communications systems often relies upon idealized antenna characteristic, where the side-lobe gain of UAVs' antenna is ignored. In practice, however, side-lobe cause inevitable interference to the ground users. We investigate the impact of UAVs' antenna side-lobe on the performance of UAVenabled communication. Our analysis shows that even for a very small antenna's side-lobe gain, the ground receiver can experience substantial interference. We further show that a rather large exclusion zone is required to ensure a sufficient level of protection for the ground receiver. Nevertheless, in a multiple-antenna setting for the ground users, even when such a large exclusion zone was in place, UAVs' antenna side-lobe creates a high level of correlation among the interference signals received across receive antennas. Such a correlation limits the system ability to exploit channel diversity in a multiple-antenna setting for improving capacity. We then quantify the impact of UAVs' antenna side-lobes on the overall system performance by deriving the corresponding loss of the achieved capacity in various communications environments. We provide a new quantitative insight on the cost of adopting non-ideal UAV antenna on the overall capacity. Our analysis also shows that the capacity loss can be confined by careful selection of system parameters. Designing UAV communication systems without considering a realistic antenna is shown to result in substantial capacity loss in real settings.
\end{abstract}

\section{INTRODUCTION}

Using Unmanned Aerial Vehicles (UAVs) equipped with wireless transceivers acting as drone base-stations (BSs) is proposed to enhance the ground users' connectivity in $4 \mathrm{G} \& 5 \mathrm{G}$ systems, and beyond [1-3]. UAVs' potential for enhancing capacity/coverage of LTE/LTE-A has also been demonstrated in cases of UAV-assisted communications and cellular-connected drones [4-6].

Adopting directional antennas at the UAVs enables direct communication with the intended ground UEs while limiting interferences to other users. Assuming an ideal directional antenna with a given beam-width and no or extremely small side-lobes, each UAV transmitter forms its own exclusive coverage zone. In this exclusive zone, the ground users are not interfered with by the UAVs whose coverage zones do not overlap. The notion of exclusion zone is adopted broadly to study the various aspects of UAV-enabled communications.

In a multiple UAV communication setting, the authors of [7] proposed a scheme for improving the spatial reuse of spectrum by selecting the proper size of the exclusion zones. Building upon the premise of exclusive coverage zone with no crosszone interference, the authors of $[8,9]$ explored the optimal positioning of UAVs in order to maximize the coverage probability. They adjust the UAVs' altitude based on the system parameters and the type of communication environment, such as sub-urban, urban, dense-urban, and high-rise. The exclusive coverage zone is also used in [10] for offloading overloaded BSs in hot-spots. [11] also investigated the coexistence of UAV and device-to-device (D2D) communications in an exclusive coverage zone. Techniques for optimal partitioning of the ground plane into exclusive coverage zones was proposed in [12], with the objective of providing coverage to all the ground users. Exclusive coverage zones were considered in [13] to support low-latency ultra-reliable UAV-enabled connectivity.

In practice, however, as also noted in [14], UAVs' antennas are not ideal. In fact, by increasing the UAVs' altitude, the ground receiver is likely to receive many interfering signals transmitted through the antenna side-lobe of other UAVs [3]. This becomes more detrimental as shown in $[5,15]$ in that for UAVs' hovering above a certain altitude, the $\mathrm{A} 2 \mathrm{G}$ propagation channel becomes dominantly Line-of-Sight (LOS) $[5,15]$. This reduces the path-loss attenuation for the interfering signals, and thus may affect the ground receivers' performance and therefore generate a substantial mismatch between theoretical and actual results. To address this issue, we investigate the effect of antenna side-lobe on the performance of ground receivers.

We consider a UAV-enabled communications system with non-ideal antennas and analyze the performance of a ground receiver with multiple receive antennas located in the exclusion zone of a given UAV. To understand the impact of the interfering signals, we then find the number of detectable interfering signals from other UAVs, or outside-UAVs, at the ground receiver, i.e., interfering signals with a power level above the ground receiver's sensitivity. Adopting tools of stochastic geometry, we find that the ground receiver is most likely to receive a rather large number of detectable interfering signals from the UAVs located outside its exclusion zone. By estimating the probability of having non-zero detectable interfering signals at the ground receiver, we introduce the required size of exclusion zone so as to keep this probability below a given performance threshold, $\epsilon$.

Our numerical evaluation shows that for antennas with a 
main-lobe to side-lobe gain ratio of $G / g=2500$ in a suburban environment, the required radius of the exclusion zone is around $50 \mathrm{~km}$ given $\epsilon=0.05$. In an extreme case of a small $G / g=12.5$, the required radius of the exclusion zone is raised to $300 \mathrm{~km}$. The size of the required exclusion zone is also affected by the communication environment. For instance, in a high-rise communication environment where the interference signals are received through a non-LOS (NLOS) dominant link, the required radius of the exclusion zone is reduced approximately by $4 \mathrm{x}$ compared to the sub-urban environment.

Interfering signals also affect the performance of multipleantennas communications between the UAV and the ground user. We show that even with an exclusion zone designed to keep the probability of having more than zero detectable interference signals higher than $\epsilon$, the aggregated received interference signal across the received antennas of the ground user becomes highly correlated. Our investigation indicates that this correlation might be as high as $90 \%$, simply due to the aggregated LOS interference induced by the signal leakage from the side-lobes of outside-UAVs. Such a correlation is detrimental to the independence of the received attending signals across the multiple-antennas in the ground user and severely affects its ability to harvest the otherwise existent channel diversity. To provide quantitative insights, we derive the expected capacity loss, and analyze the impact of various system parameters on such a capacity loss. Our analysis shows that unlike the terrestrial communications in which non-LOS propagation is often dominant, designing UAV communication systems without considering a realistic antenna pattern might lead to a substantial capacity loss in real settings.

\section{System MODEL}

We consider a ground user located at the origin receiving signals from a UAV, namely a supporting $U A V$, located exactly above the origin at altitude $\tilde{H}>0$. Associated with this transmitter-receiver link, we also consider an exclusion zone, where the receiver is assumed to be located at its center. To protect the ground receiver against interferences, other UAVs hovering above the inclusion zone, namely outside$U A V S$, are not allowed to transmit. Relevant to our analysis is the projection of the exclusion zone on the ground which is a circular disk with radius $Z>0$. We denote the exclusion zone (resp. outside of the exclusion zone) by a disk, $\mathcal{B}_{O}(Z)$ (resp. annulus $\overline{\mathcal{B}}_{O}(Z)$ ).

We assume that the locations of the outside-UAVs in the 3D space follow a Homogenous Poisson Point Process (HPPP). In this model, $\Phi=\left\{\left(X_{i}, H\right) \in \mathbb{R}^{3}, i=1,2, \ldots:\left\|X_{i}\right\|>\right.$ $Z\}$, where $X_{i} \in \overline{\mathcal{B}}_{O}(Z)$ is the location of UAV $i$ in the 2$\mathrm{D}$ plane, and $H$ is its altitude. For brevity, we further assume that the UAVs are all at the same constant altitude. The density of UAVs is $\lambda$ units per $\mathrm{km}^{2}$. All UAVs are equipped with a directional antenna with a circular radiation pattern and beamwidth of $\omega$. We denote the main-lobe and side-lobe antenna gain for UAV $X_{i}$ by $X_{i}$, and $g \neq 0$, respectively, where $g \ll$ $G$. The vertical angel between the receiver and the UAV $X_{i}$ is also denoted by $\psi_{i}=\tan ^{-1}\left(H /\left\|X_{i}\right\|\right)$. The receiver located at the origin is within the main-lobe of UAV $i$, if $\psi_{i}>\pi / 2-$ $\omega / 2$, or equivalently for $\left\|X_{i}\right\|<\frac{H}{\tan (\pi / 2-\omega / 2)}$. Therefore, to ensure that the receiver does not receive interference from the main-lobe of UAV $X_{i}, Z$ should be set to $Z=\frac{H}{\tan (\pi / 2-\omega / 2)}$. Therefore, the exclusion zone of $X_{i}$ is not in the main-lobe of the out-side UAVs.

TABLE I

Air-TO-GRound CHANNEl PARAMETERS [8].

\begin{tabular}{|c|cccc|}
\hline & High-Rise & Dense-Urban & Urban & Sub-Urban \\
\hline$\phi$ & 27.23 & 12.08 & 9.61 & 4.88 \\
$\psi$ & 0.08 & 0.11 & 0.16 & 0.43 \\
\hline
\end{tabular}

The channel between the UAVs and the users on the ground, referred to as the $A 2 G$ channel, is modeled as a combination of a large-scale path-loss attenuation and a small-scale fading component $[9,15]$. The A2G channel operates in LOS/NLOS mode [9], and the occurrence of LOS mode is shown to be dependent, among other things, on the drone's height, elevation angle, and the type of communication environment, e.g., dense urban or sparse rural. The probability that the channel between UAV $X_{i}$ and the receiver is an LOS-dominant channel is often considered as the distance-dependent probability $[8,9]$ :

$$
p_{L}\left(\left\|X_{i}\right\|\right)=\left(1+\phi e^{-\psi\left(\frac{180}{\pi} \arctan \left(\frac{H}{\left\|X_{i}\right\|}\right)-\phi\right)}\right)^{-1},
$$

where $\left\|X_{i}\right\|$ is the 2-D Euclidian distance between the ground user and the drone $X_{i}$, and $\phi$ and $\psi$ are the channel parameters representing the characteristics of the communication environment. As shown in (1), the probability of experiencing an LOS-dominant channel is increased by increasing $H$. Using (1), the path-loss attenuation is:

$$
L\left(\left\|X_{i}\right\|\right)= \begin{cases}L_{L}\left(\left\|X_{i}\right\|\right)=\frac{K_{L}}{\left(\sqrt{H^{2}+\left\|X_{i}\right\|}\right)^{\alpha}} & \sim p_{L}\left(\left\|X_{i}\right\|\right), \\ L_{N}\left(\left\|X_{i}\right\|\right)=\frac{K_{N}}{\left(\sqrt{H^{2}+\left\|X_{i}\right\|}\right)^{\alpha}} & \sim p_{N}\left(\left\|X_{i}\right\|\right) .\end{cases}
$$

where $\alpha_{L}\left(\alpha_{N}\right)$ is the LOS (NLOS) path-loss exponent, and $K_{L}\left(K_{N}\right)$ is the corresponding intercept constant, $\alpha_{L} \ll \alpha_{N}$. Note that increasing $H$ results in a higher attenuation as the signal needs to travel farther, experiencing a greater power loss, or equivalently a higher transmission power is required by the UAVs. Nevertheless, since $\alpha_{L} \ll \alpha_{N}$, a larger $H$ might be advantageous as it may make the LOS component dominant. In practice, $H$ should be carefully designed to balance the required transmit power on one hand, and the channel attenuation advantage on the other hand, see, e.g., $[8,9]$.

Each receiver has $R \geq 2$ antennas, indexed by $r$. Smallscale power fading between UAV $X_{i}$ and the $r$-th antenna at the receiver, $V_{X_{i}, r}$ is modeled by Nakagami fading:

$$
V_{X_{i}, r}= \begin{cases}V_{X_{i}, r}^{L}=\Gamma\left(\bar{V}_{L}, \frac{1}{\bar{V}_{L}}\right) & \sim p_{L}\left(\left\|X_{i}\right\|\right) \\ V_{X_{i}, r}^{N}=\Gamma\left(\bar{V}_{N}, \frac{1}{\bar{V}_{N}}\right) & \sim p_{N}\left(\left\|X_{i}\right\|\right),\end{cases}
$$

where $\Gamma(a, b)$ is Gamma distribution with parameters $a$ and $b$. For UAV $X_{i}$, we assume that $V_{X_{i}, r}$ are independent $\forall r$. Parameters $a$ and $b$ depend on the LOS/NLOS status of the communication channel between UAV $X_{i}$ and the receiver. We 
also note the fading is often more severe in NLOS channels, so it is reasonable to assume $\bar{V}_{L}>\bar{V}_{N}$.

\section{Count of Interfering Signals}

Ideally, the side-lobe is NULL, i.e., $g=0$, the receiver does not detect any signal from outside-UAVs. This simplifying assumption is widely adopted in the related literature, see, e.g., $[7,9]$. In practice, however, $g \neq 0$, but the antenna is designed such that $g / G \ll 1$. Therefore, the signal transmitted by the outside-UAVs through the side-lobes may cause interference at the receiver. To investigate the impact of antenna sidelobes, we obtain the number of detectable interfering outsideUAVs signals at the receiver, or signal count, $\Sigma$. A similar terminology is used in the LTE systems, where the signal count to quantify the interference of neighboring cells [3].

We evaluate the signal count based on the aggregated received power across receive antennas,

$$
\Sigma=\sum_{X_{i} \in \Phi} 1\left(\sum_{r} g P L\left(\left\|X_{i}\right\|, H\right) V_{X_{i}, r} \geq \gamma\right),
$$

where $\gamma>0$ is the receiver's sensitivity.

We now show that the signal count $\Sigma$ is (4) is a Poisson random variable. For this, we derive its Laplace transform:

$$
\begin{gathered}
\mathcal{L}_{\Sigma}(t)=\mathbb{E} e^{-t} \sum_{X_{i} \in \Phi} 1\left(\sum_{r} g P L\left(\left\|X_{i}\right\|, H\right) V_{X_{i}, r} \geq \gamma\right) \\
=\mathbb{E} \prod_{X_{i} \in \Phi}\left(e^{-t} \mathbb{P}\left\{\sum_{r} V_{X_{i}, r} \geq \frac{\gamma}{g P L\left(\left\|X_{i}\right\|, H\right)}\right\}\right. \\
\left.+\mathbb{P}\left\{\sum_{r} V_{X_{i}, r}<\frac{\gamma}{g P L\left(\left\|X_{i}\right\|, H\right)}\right\}\right) \\
=\mathbb{E}_{\Phi} \prod_{X_{i} \in \Phi}\left(\sum _ { l _ { i } \in \{ L , N \} } p _ { l _ { i } } ( y , H ) \left(e ^ { - t } \mathbb { P } \left\{\sum_{r} V_{X_{i}, r}^{l_{i}} \geq\right.\right.\right.
\end{gathered}
$$

(a):Sub-Urban

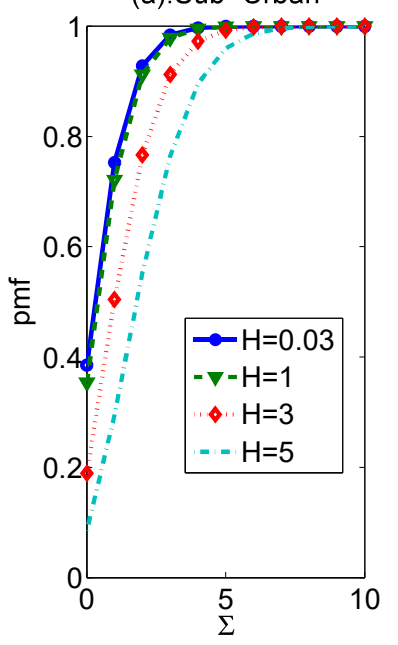

(b):High-Rise

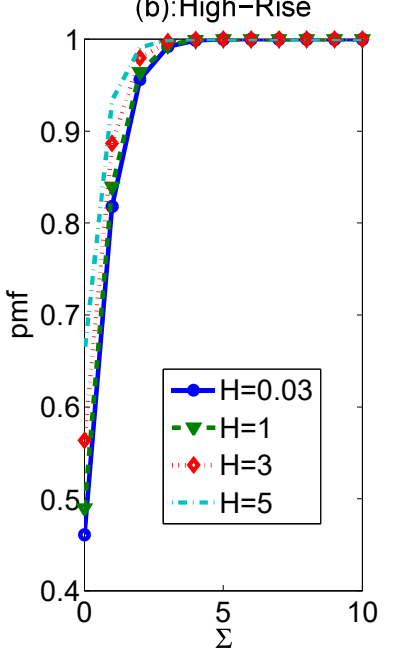

Fig. 1. The probability mass function (pmf) of the interference signal count, $\Sigma$, for $\lambda=10^{-3}, G / g=2500$, and $\gamma=10^{-8}$.

$$
\begin{aligned}
& \left.\left.\left.\frac{\gamma}{g P L\left(\left\|X_{i}\right\|, H\right)}\right\}+\mathbb{P}\left\{\sum_{r} V_{X_{i}, r}^{l_{i}}<\frac{\gamma}{g P L\left(\left\|X_{i}\right\|, H\right)}\right\}\right)\right) \\
& =e^{-2 \pi \lambda\left(1-e^{-t}\right)} \sum_{l \in\{L, N\}} \int_{Z}^{\infty} y p_{l}(y, H) \mathbb{P}\left\{\sum_{r} V_{y, r}^{l} \geq \frac{\gamma}{g P L_{l}(y, H)}\right\} \\
& d
\end{aligned}
$$

where in the second step, the LOS/NLOS status of communication links are independently drawn, and the last step is due to the Laplace generation functional (LGFL) of PPP. Using (5), the signal count is confirmed to be a Poisson random variable with the mean value:

$$
\bar{\Sigma}=2 \pi \lambda \sum_{l \in\{L, N\}} \int_{Z}^{\infty} y p_{l}(y, H) \bar{F}_{\sum_{n} V_{n}^{l}}\left(\frac{\gamma}{g P L_{l}(y, H)}\right) \mathrm{d} y .
$$

Obtaining $\bar{\Sigma}$ in (6) requires the complementary cumulative distributed function (CCDF) of random variable, $\sum V_{r}^{l}$, namely $\bar{F}_{\sum_{r} V_{r}^{l}}($.$) . We then assume \sum_{r} V_{r}^{l} \geq N \min _{r} V_{r}^{l}$ and further note that the fading power gains across antennas are i.i.d. normalized Gamma random variables, and therefore

$$
\begin{aligned}
& \bar{F}_{\sum_{n} V_{n}^{l}}\left(\frac{\gamma}{g P L_{l}(y, H)}\right) \geq\left(\bar{F}_{V_{1}^{l}}\left(\frac{\gamma}{N g P L_{l}(y, H)}\right)\right)^{N} \\
& =e^{-\frac{\gamma \bar{V}_{l}}{g P L_{l}(y, H)}}\left(1-\sum_{m=0}^{\bar{V}_{l}} \frac{1}{m !} \frac{\bar{V}_{l}^{m} \gamma^{m}}{\left(N g P L_{l}(y, H)\right)^{m}}\right)^{N} .
\end{aligned}
$$

An upper-bound on $\bar{F}_{\sum_{n} V_{n}^{l}}($.$) is then obtained using (6), (7).$

We now use the approximated distribution of $\Sigma$ to study the (cumulative) pmf, i.e., $F_{\Sigma}(v)=\sum_{s=0}^{v} \frac{\bar{\Sigma}^{s}}{s !} e^{-\bar{\Sigma}}$. This quantity measures the probability that the signal count is smaller than a given number, and is plotted in Fig. 1 for high-rise and sub-urban environments. For a given receiver, it is highly likely to receive a large number of interfering signals. So, even where the antenna side-lobe gain is relatively very small, $G / g \gg 1$, since an exclusion zone fails to protect the ground receiver against the interference. Fig. 1 further shows that increasing the altitude of the outside-UAVs makes two completely different impacts on the pmf in the two communication environments: increasing $H$ reduces (increases) pmf in the sub-urban (high-rise) environment, i.e., $H$ increases due to multi-path dominance in the high-rise environment.

In practice, if $\Sigma$ becomes greater than a given threshold number, the ground receiver might report to the network. To preserve the receive quality, the network may adopt a combination of actions to mitigate/reduce the received interference. Here we assume that there is no such mechanism available, and instead attempt to gain quantitative insight on the design of the required size of exclusion zone, $Z^{*}$, that guarantees all outside-UAVs to be invisible to the receiver.

Let $O_{Z}$ denote the probability of $\Sigma>0$. Our objective is then to evaluate $Z^{*}$, such that $O_{Z} \geq \epsilon$, where $\epsilon \in(0,1)$ is a 
(a): High Rise

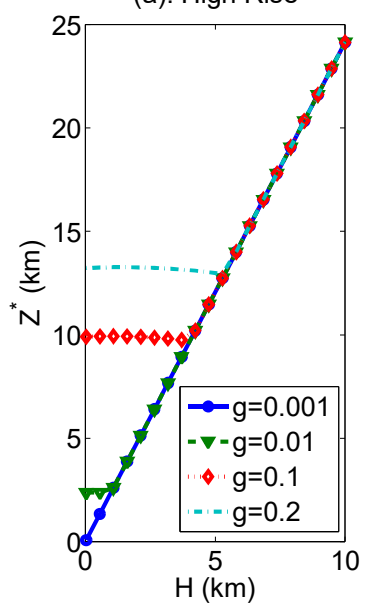

(b): Dense Urban

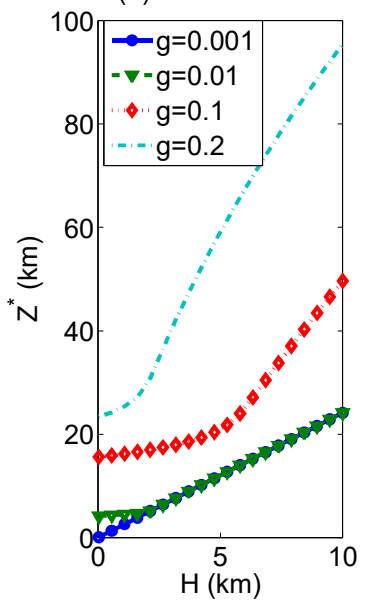

(c): Urban

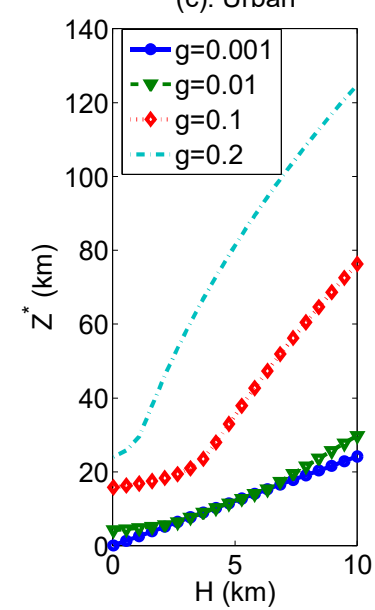

(d): Sub-Urban

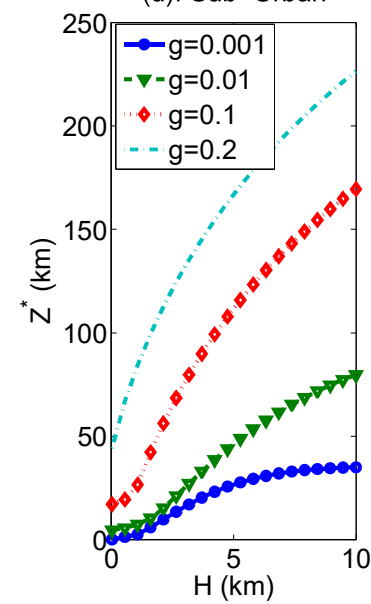

Fig. 2. Value of $Z^{*}$ versus the altitude of UAVs, where $\lambda=10^{-3}$, and $\epsilon=0.05$.

system parameter. Using (7), we write

$$
\begin{gathered}
O_{Z} \leq \exp \left\{-2 \pi \lambda \sum_{l \in\{L, N\}} \int_{Z}^{\infty} y p_{l}(y, H) e^{-\frac{\gamma \bar{V}_{l}}{g P L_{l}(y, H)}}\right. \\
\left.\left(1-\sum_{m=0}^{\bar{V}_{l}} \frac{1}{m !} \frac{\bar{V}_{l}^{m} \gamma^{m}}{\left(N g P L_{l}(y, H)\right)^{m}}\right)^{N} \mathrm{~d} y\right\} .
\end{gathered}
$$

Setting the upper-bound equal to $\epsilon, Z^{*}$ is obtained via the following equation

$$
\begin{gathered}
\sum_{l \in\{L, N\}} \int_{Z^{*}}^{\infty} \frac{y p_{l}(y, H)}{e^{\frac{\gamma \bar{V}_{l}}{g P L_{l}(y, H)}}}\left(1-\sum_{m=0}^{\bar{V}_{l}} \frac{1}{m !} \frac{\bar{V}_{l}^{m} \gamma^{m}}{\left(N g P L_{l}(y, H)\right)^{m}}\right)^{N} \mathrm{~d} y \\
=\frac{\log (1 / \epsilon)}{2 \pi \lambda} .
\end{gathered}
$$

Fig. 2 plots $Z^{*}$ versus the altitude of interfering UAVs, $H$, in prevalent communication environments for several values of antenna side-lobe gain, $g$, where $\epsilon=0.05$. Increasing the side-lobe gain, $g$, substantially increases $Z^{*}$. Fig. 2 also shows that even for a very small $g$, a rather large exclusion zone is required. The size of exclusion zone is also increased by increasing the UAVs' altitude.

Among the various type of communication environments, unlike the high-rise case, the sub-urban environment requires a substantially large exclusion zone, almost $4 x$ larger as Fig. 2 shows, due mainly to the dominance of LOS component in the sub-urban environment.

\section{IMPACT ON THE CAPACITY}

Setting the size of exclusion zone based on the results in Section II reduces the negative impact of non-ideal antennas, i.e., $g \neq 0$, and limits the likelihood of introducing interfering signals at the receiver.

The performance of a multi-antenna receiver can also degrade due to the cross-antenna signal correlation. For the ideal case of $g=0$, no cross-antenna signal correlation exists as the fading across antennas are independent. This enables the receive array to harness the diversity of the wireless channels and achieve diversity and/or multiplexing gains. In practice, where $g \neq 0$, the aggregated interference caused by the sidelobe induce cross-antenna signal correlation even when the exclusion zone $B_{O}\left(Z^{*}\right)$ is considered. In what follows, we investigate the capacity loss at the receiver because of the existing correlation in the received signal across the receive antennas.

\section{A. Cross-Antenna Interference Correlation}

The cross-antenna interference correlation coefficient is:

$$
\rho_{r, r^{\prime}}=\frac{\mathbb{E}\left[I_{r} I_{r^{\prime}}\right]-\mathbb{E}\left[I_{r}\right] \mathbb{E}\left[I_{r^{\prime}}\right]}{\sqrt{\operatorname{Var}\left(I_{r}\right) \operatorname{Var}\left(I_{r^{\prime}}\right)}}=\frac{\mathbb{E}\left[I_{r} I_{r^{\prime}}\right]-\left(\mathbb{E}\left[I_{r^{\prime}}\right]\right)^{2}}{\operatorname{Var}\left(I_{r}\right)},
$$

where the received interference at antenna $r$ is

$$
I_{r}=\sum_{X_{i} \in \Phi} P L\left(\left\|X_{i}\right\|, H\right) g V_{X_{i}, r} .
$$

Proposition 1: The cross-antenna interference correlation coefficient is

$$
\rho_{r, r^{\prime}}=\frac{\sum_{l \in\{\mathrm{L}, \mathrm{N}\}} W_{l}}{\sum_{l \in\{\mathrm{L}, \mathrm{N}\}} \frac{\bar{V}_{l}+1}{\bar{V}_{l}} W_{l}}=\frac{1}{1+\frac{\sum_{l \in\{\mathrm{L}, \mathrm{N}\}} \frac{W_{l}}{\bar{V}_{l}}}{\sum_{l \in\{\mathrm{L}, \mathrm{N}\}} W_{l}}},
$$

where $r \neq r^{\prime}$, and

$$
W_{l}=\int_{Z^{*}}^{\infty} x p_{l}(x, H)\left(L_{l}(x, H)\right)^{2} \mathrm{~d} x .
$$

Proof: See the Appendix.

For any positive real numbers, $\left\{a_{m}, b_{m}\right\}, m=1,2, \ldots$, it is easy to show that $\sum_{m} \frac{a_{m}}{b_{m}} \geq \frac{\sum_{m} a_{m}}{\sum_{m} b_{m}}$. Therefore, $\frac{\sum_{l \in\{\mathrm{L}, \mathrm{N}\}} \frac{W_{l}}{\bar{V}_{l}}}{\sum_{l \in\{\mathrm{L}, \mathrm{N}\}} W_{l}} \leq$ 
(a): High Rise

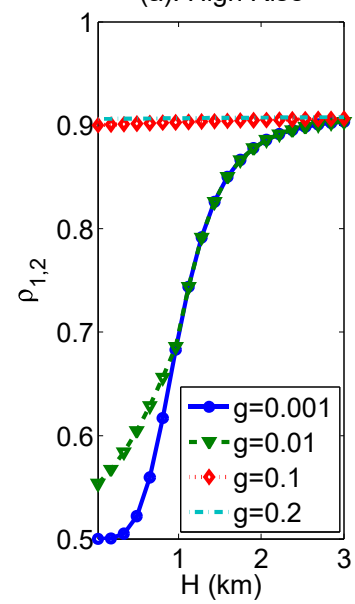

(b): Dense Urban

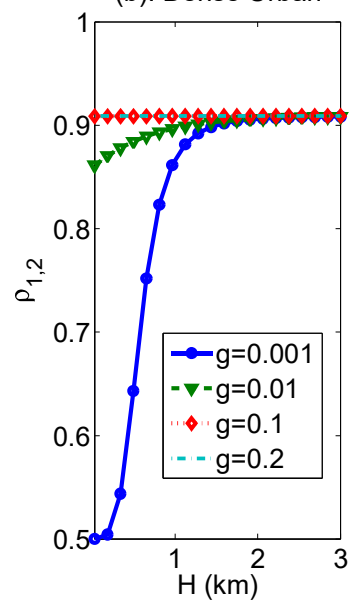

(c): Urban

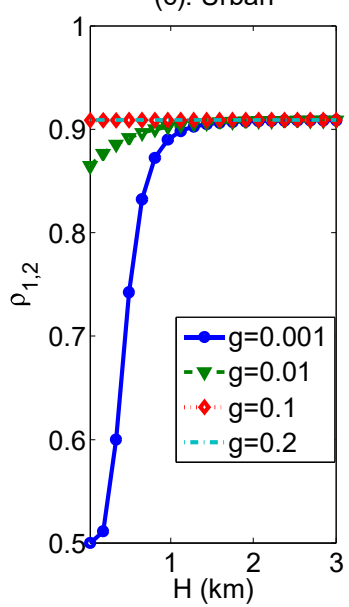

(d): Sub-Urban

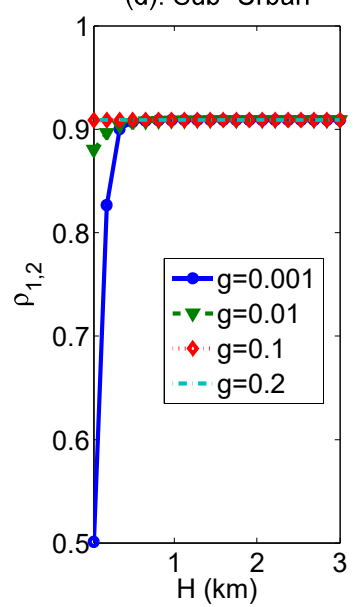

Fig. 3. Cross-antenna interference correlation coefficient versus the altitude of UAVs, where $\lambda=10^{-3}$.

$\sum_{l \in\{\mathrm{L}, \mathrm{N}\}} \frac{1}{\bar{V}_{l}}$. This suggests a lower-bound on $\rho_{r, r^{\prime}}$ as $\rho_{r, r^{\prime}} \geq$ $\frac{1}{1+\sum_{l \in\{\mathrm{L}, \mathrm{N}\}} \frac{1}{\bar{V}_{l}}}$. An upper-bound on $\rho_{r, r^{\prime}}$ is also obtained as

$$
\rho_{r, r^{\prime}} \leq \sum_{l \in\{\mathrm{L}, \mathrm{N}\}} \frac{1 W_{l}}{\bar{V}_{l}+1} \frac{\sum_{l} W_{l}}{\bar{V}_{l}}=\sum_{l \in\{\mathrm{L}, \mathrm{N}\}} \frac{\bar{V}_{l}}{\bar{V}_{l}+1} .
$$

Therefore,

$$
\frac{1}{1+\sum_{l \in\{\mathrm{L}, \mathrm{N}\}} \frac{1}{\bar{V}_{l}}} \leq \rho_{r, r^{\prime}} \leq \sum_{l \in\{\mathrm{L}, \mathrm{N}\}} \frac{1}{1+\frac{1}{\bar{V}_{l}}} .
$$

Using the above, for a Rayleigh distributed fading in the NLOS link, it is easy to show $\rho_{r, r^{\prime}} \gtrsim 0.5$.

Fig. 3 plots $\rho_{1,2}$ versus $\tilde{H}$, showing a considerable level of interference correlation, $\rho_{1,2} \geq 0.5$, despite consideration of exclusion zone to protect the receiver from the direct interference received from the outside-UAVs. Such a correlation limits the achievable gain of the receive diversity in the $\mathrm{A} 2 \mathrm{G}$ communication link. Increasing $g$ also leads to a substantial increase of $\rho_{1,2}$. Fig. 3 further highlights the impact of the communication environment. A much higher antenna correlation is experienced in the sub-urban environment due to the dominance of the LOS interference.

\section{B. Capacity Loss}

The supporting UAV, denoted by $X_{0}$, is located at height $\tilde{H}$ on top of the receiver. We assume that the same channel model discussed in Section II is valid for the communication link between the supporting UAV and the ground receiver. The achievable rate of the receiver is denoted by $C(g)$.

We evaluate $\Delta_{R}=C(0)-C(g)$ for a given system with an exclusion zone of $B_{O}\left(Z^{*}\right)$. Assuming maximal ratio combining, which is optimal for the case of no interference [16], $\Delta_{R}$ is then formulated as

$$
\Delta_{R}=\mathbb{E}\left[\log \left(1+\sum_{r=1}^{R} \frac{P L\left(\left\|X_{0}\right\|, \tilde{H}\right) G V_{X_{0}, r}}{\sigma^{2}}\right)-\right.
$$

$$
\begin{aligned}
& \log \left(1+\sum_{r=1}^{R} \frac{\left.P L\left(\left\|X_{0}\right\|, \tilde{H}\right) G V_{X_{0}, r}\right)}{\sigma^{2}+I_{r}}\right] \\
\approx & \mathbb{E}\left[\log \left(1+\frac{P L\left(\left\|X_{0}\right\|, \tilde{H}\right) G \sum_{r=1}^{R} V_{X_{0}, r}}{\sigma^{2}}\right)-\right.
\end{aligned}
$$

$$
\left.\log \left(1+\frac{P L\left(\left\|X_{0}\right\|, \tilde{H}\right) G \sum_{r=1}^{R} V_{X_{0}, r}}{\sigma^{2}+\hat{I}}\right)\right]
$$

where $\sigma^{2}$ is the AWGN power, the interference received at each antenna is approximated as $\hat{I}=\sum_{X_{i} \in \Phi} P L\left(\left\|X_{i}\right\|, H\right) g V_{X_{i}}$, and $V_{X_{i}}$ is distributed as in (3) and does not depend on the antenna index. This is due to highly correlated interference across antennas, as substantiated in Proposition 1.

Using $\log (1+x)=\int_{0}^{\infty} e^{-v} / v\left(1-e^{-v x}\right) \mathrm{d} v$, we then write $\Delta_{R}$ as:

$$
\Delta_{R} \approx \int_{0}^{\infty} e^{-v \sigma^{2}} / v\left(1-\mathcal{L}_{\hat{I}}(v)\right) \mathcal{L}_{S}(v) \mathrm{d} v
$$

where $\mathcal{L}_{\hat{I}}(v)$ is the Laplace transform of $\hat{I}$,

$$
\begin{aligned}
& \mathcal{L}_{\hat{I}}(v)=\mathbb{E}\left[e^{-v \hat{I}}\right]=\mathbb{E}_{\Phi} \prod_{X_{i} \in \Phi} \mathbb{E} e^{-v g P L\left(\left\|X_{i}\right\|, H\right) V_{X_{i}}} \\
= & \mathbb{E}_{\Phi} \prod_{X_{i} \in \Phi}\left(\sum_{l_{i} \in\{L, N\}} \frac{p_{l_{i}}\left(\left\|X_{i}\right\|, H\right)}{\left(1+v P g L_{l_{i}}\left(\left\|X_{i}\right\|, H\right) / \bar{V}_{l_{i}}\right)^{\bar{V}_{l_{i}}}}\right) \\
= & e^{-2 \pi \lambda \sum_{l \in\{L, N\}} \int_{Z^{*}}^{\infty} y p_{l}(y, H)\left(1-\left(1+v P g L_{l}(y, H) / \bar{V}_{l_{i}}\right)^{-\bar{V}_{l}}\right) \mathrm{d} y} .
\end{aligned}
$$


In (12), $\mathcal{L}_{S}(v)$ is the Laplace transform of the effective received power from the supporting UAV $X_{0}$ :

$$
\mathcal{L}_{S}(v)=\sum_{l \in\{L, N\}} \frac{p_{l}(0, \tilde{H})}{\left(1+v G P K_{l} \tilde{H}^{-\alpha_{l}}\right)^{\bar{V}_{l}}} .
$$

Fig. 4 plots $\Delta_{R}$ versus $H$ for several values of $g$ in different communication environments, showing that the loss of capacity in the high-rise environment is the lowest. Fig. 4a also demonstrates that for $g \geq 0.1$, increasing $H$ results in a higher capacity loss, $\Delta_{R}$ because of a higher chance of experiencing interference through LOS dominant links. For $g<0.1$, however, increasing $H$ reduces the capacity loss. This is partly because there is no dominant LOS interference. Existence of the exclusion zone also reduces the effect of NLOS interfering links. Figs. 4-b and 4-c also show that capacity loss is higher in the urban environment than the dense-urban.

For the case of sub-urban, Fig. 4-d suggests that for $g \leq 0.1$, the highest capacity loss is experienced at a certain UAVs' altitude. Also for $H \approx 1$, a slightly larger capacity loss is experienced for $g=0.1$ than for $g=0.2$. This is mainly due to a very large exclusion zone when $g=0.2$ (see Fig. 2-d). In fact, a combination of factors including the likelihood of receiving LOS interference, higher path-loss attenuation, and the size of exclusion zone affect the capacity loss. Depending on this combination, the capacity loss may increase or decrease by increasing $H$.

\section{Conclusions}

We have investigated the impact of UAVs' antenna sidelobe on the performance of UAV-enabled communication. Our analysis and results demonstrated that even for a very small value of the antenna's side-lobe gain, the ground receiver can experience substantial interference. We further showed that a rather large exclusion zone is required to ensure a sufficient level of protection for the ground receiver. Nevertheless, in a multiple-antenna setting for the ground users, even when such large exclusion zone was in place, UAVs' antenna sidelobe creates a high level of correlation among the interference signals received across receive antennas. Such a correlation among the received signals limits the system's ability to exploit channel diversity in a multiple-antenna setting for improving capacity. Using these results/observations, we then quantified the impact of UAVs' antenna side-lobes on the overall system performance by obtaining the corresponding loss on the achieved capacity in various communications environments. We observed that the capacity loss can be limited by careful selection of system parameters. Finally, our results indicate that unlike the terrestrial communications in which non-LOS propagation is often dominant, designing UAV communication systems without considering a realistic antenna pattern might lead to substantial capacity loss in real settings.
Appendix: Proof of Proposition 1

Using Campbell-Mecke Theorem [17],

$$
\begin{gathered}
\mathbb{E}\left[I_{r}\right]=g \mathbb{E} \sum_{X_{i} \in \Phi} P L\left(\left\|X_{i}\right\|, H\right) V_{X_{i}, r} \\
=2 \pi P g \lambda \int_{Z^{*}}^{\infty} x \mathbb{E}\left[L(x, H) V_{x, r}\right] \mathrm{d} x \\
=2 \pi g P \lambda \sum_{l \in\{\mathrm{L}, \mathrm{N}\}} \int_{Z^{*}}^{\infty} x p_{l}(x, H) L_{l}(x) \mathrm{d} x,
\end{gathered}
$$

where we note $\mathbb{E}\left[V_{l}\right]=1$. Further, for $r \neq r^{\prime}$

$$
\begin{gathered}
\mathbb{E}\left[I_{r} I_{r^{\prime}}\right]=g^{2} P^{2} \mathbb{E} \sum_{X_{i} \in \Phi} \sum_{X_{j} \in \Phi} L\left(\left\|X_{i}\right\|, H\right) \\
\times L\left(\left\|X_{j}\right\|, H\right) V_{X_{i}, r} V_{X_{j}, r^{\prime}} \\
=g^{2} P^{2} \mathbb{E} \sum_{X_{i} \in \Phi}\left(L\left(\left\|X_{i}\right\|, H\right)\right)^{2} V_{X_{i}, r} V_{X_{i}, r^{\prime}} \\
+g^{2} P^{2} \mathbb{E} \sum_{X_{i} \in \Phi} \sum_{X_{j} \in \Phi \backslash X_{i}} L\left(\left\|X_{i}\right\|, H\right) L\left(\left\|X_{j}\right\|, H\right) V_{X_{i}, r} V_{X_{j}, r^{\prime}} \\
=2 \pi g^{2} P^{2} \lambda \sum_{l \in\{\mathrm{L}, \mathrm{N}\}} \bar{V}_{l}^{2} \int_{Z^{*}}^{\infty} x p_{l}(x, H)\left(L_{l}(x, H)\right)^{2} \mathrm{~d} x \\
+4 \pi^{2} g^{2} P^{2} \lambda^{2} \sum_{l \in\{\mathrm{L}, \mathrm{N}\}} \sum_{l^{\prime} \in\{\mathrm{L}, \mathrm{N}\}} \bar{V}_{l} \bar{V}_{l^{\prime}} \int_{Z^{*}}^{\infty} \int_{Z^{*}}^{\infty} x_{1} x_{2} \\
p_{l}\left(x_{1}, H\right) L_{l}\left(x_{1}, H\right) p_{l^{\prime}}\left(x_{2}, H\right) L_{l^{\prime}}\left(x_{2}, H\right) \mathrm{d} x_{1} \mathrm{~d} x_{2} \\
=2 \pi g^{2} P^{2} \lambda \sum_{l \in\{\mathrm{L}, \mathrm{N}\}} \int_{Z^{*}}^{\infty} x p_{l}(x, H)\left(L_{l}(x, H)\right)^{2} \mathrm{~d} x \\
+4 \pi^{2} g^{2} P^{2} \lambda^{2} \sum_{l \in\{\mathrm{L}, \mathrm{N}\}} \sum_{l^{\prime} \in\{\mathrm{L}, \mathrm{N}\}} \int_{Z^{*}}^{\infty} \int_{Z^{*}}^{\infty} x_{1} x_{2} \\
p_{l}\left(x_{1}, H\right) L_{l}\left(x_{1}, H\right) p_{l^{\prime}}\left(x_{2}, H\right) L_{l^{\prime}}\left(x_{2}, H\right) \mathrm{d} x_{1} \mathrm{~d} x_{2} .
\end{gathered}
$$

Using (15), and (14), it is easy to show

$$
\begin{gathered}
\mathbb{E}\left[I_{r} I_{r^{\prime}}\right]-\left(\mathbb{E}\left[I_{r^{\prime}}\right]\right)^{2}= \\
2 \pi g^{2} P^{2} \lambda \sum_{l \in\{\mathrm{L}, \mathrm{N}\}} \int_{Z^{*}}^{\infty} x p_{l}(x, H)\left(L_{l}(x, H)\right)^{2} \mathrm{~d} x .
\end{gathered}
$$

Similarly, noting that $\mathbb{E}\left[\left(V_{l}\right)^{2}\right]=\frac{\bar{V}_{l}+1}{\bar{V}_{l}}$,

$$
\operatorname{Var}\left(I_{r}\right)=2 \pi g^{2} P^{2} \lambda \sum_{l \in\{\mathrm{L}, \mathrm{N}\}} \frac{\bar{V}_{l}+1}{\bar{V}_{l}} W_{l} .
$$

Substituting (16) and (17) into the definition of crossantenna correlation in (8) completes the proof. 
(a): High Rise

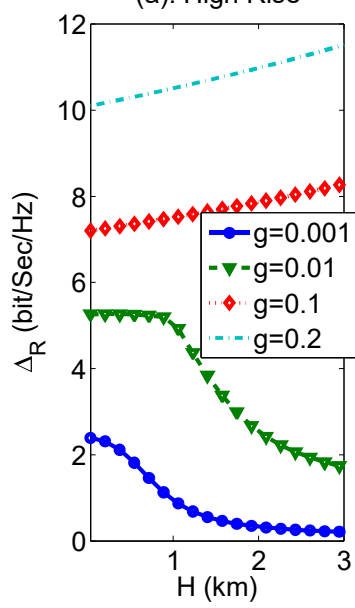

(b): Dense Urban

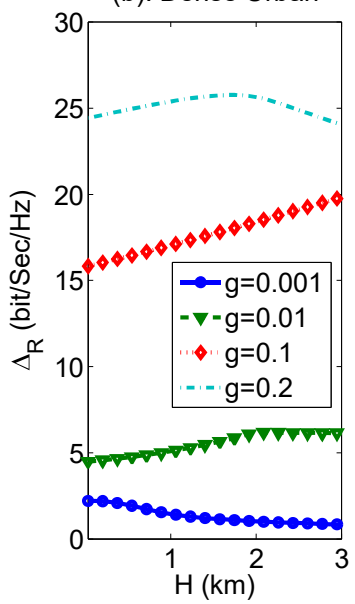

(c): Urban

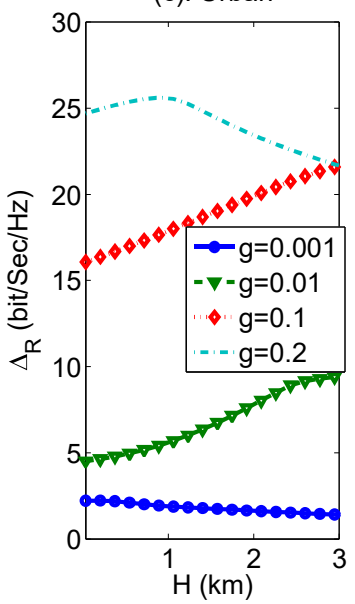

(d): Sub-Urban

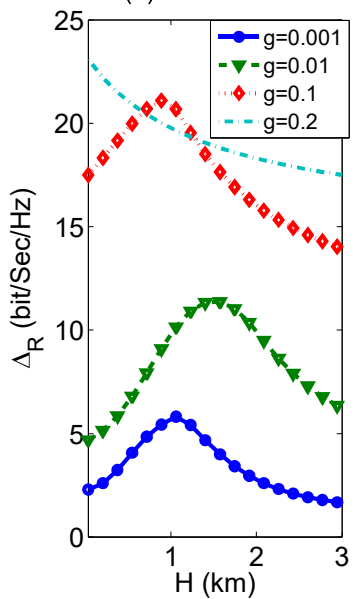

Fig. 4. Capacity loss versus $H$, where $\tilde{H}=300 \mathrm{~m}$, and $\lambda=10^{-3}$.

\section{REFERENCES}

[1] S. Sekander, H. Tabassum, and E. Hossain, "Multi-tier drone architecture for $5 \mathrm{G} / \mathrm{B} 5 \mathrm{G}$ cellular networks: Challenges, trends, and prospects," IEEE Commun. Mag., vol. 56, no. 3, pp. 96-103, Mrc. 2018.

[2] Y. Zeng, R. Zhang, and T. J. Lim, "Wireless communications with unmanned aerial vehicles: opportunities and challenges," IEEE Commun. Mag., vol. 54, no. 5, pp. 36-42, May 2016.

[3] X. Lin, V. Yajnanarayana, S. D. Muruganathan, S. Gao, H. Asplund, H.L. Maattanen, M. Bergstrom, S. Euler, and Y.-P. E. Wang, "The sky is not the limit: LTE for unmanned aerial vehicles," IEEE Commun. Mag., vol. 56, no. 4, pp. 204-210, Apr. 2018.

[4] Qualcomm, "LTE unmanned aircraft systemstrial report," Qualcomm Technologies, Inc., May 2017.

[5] R1-1705823, "Field measurement results for drone LTE enhancement," in 3GPP TSG-RAN WG1 Meeting 88bis. KDDI Corporation, Mar. 2017.

[6] RP-170779, "New SID on enhanced support for aerial vehicles," in 3GPP TSG RAN Meeting 75. NTT DOCOMO INC, Mar. 2017.

[7] J. Lyu and R. Zhang, "Blocking probability and spatial throughput characterization for cellular-enabled UAV network with directional antenna," 2017, [Online]. Available: https://arxiv.org/pdf/1710.10389.pdf.

[8] A. Al-Hourani, S. Kandeepan, and S. Lardner, "Optimal lap altitude for maximum coverage," IEEE Wireless Commun. Letters, vol. 3, no. 6, pp. 569-72, Dec. 2014.

[9] R. I. Bor-Yaliniz, A. El-Keyi, and H. Yanikomeroglu, "Efficient 3-D placement of an aerial base station in next generation cellular networks," in Proc. IEEE ICC, Kuala Lumpur, Malaysia, May 2016.
[10] J. Lyu, Y. Zeng, and R. Zhang, "UAV-aided offloading for cellular hotspot," IEEE Trans. Wireless Commun., vol. 17, no. 6, pp. 3988-4000, Jun. 2018.

[11] E. Turgut and M. C. Gursoy, "Downlink analysis in unmanned aerial vehicle (UAV) assisted cellular networks with clustered users," IEEE Access, vol. 6, pp. 36313-36324, 2018.

[12] M. Mozaffari, W. Saad, M. Bennis, and M. Debbah, "Wireless communication using unmanned aerial vehicles (UAVs): Optimal transport theory for hover time optimization," IEEE Trans. Wireless Commun., vol. 16, no. 12, pp. 8052-8066, Dec. 2017.

[13] C. She, C. Liu, T. Q. S. Quek, C. Yang, and Y. Li, "UAV-assisted uplink transmission for ultra-reliable and low-latency communications," in proc. IEEE ICC Workshop, Dec. 2018.

[14] S. D. Muruganathan, X. Lin, H.-L. Maattanen, Z. Zou, W. A. Hapsari, and S. Yasukawa, "An overview of 3GPP release-15 study on enhanced LTE support for connected drones," 2018, [Online]. Available: https://arxiv.org/abs/1805.00826.

[15] B. van der Bergh, A. Chiumento, and S. Pollin, "LTE in the sky: trading off propagation benefits with interference costs for aerial nodes," IEEE Commun. Mag., vol. 54, no. 5, pp. 44-50, May 2016.

[16] R. Tanbourgi, H. S. Dhillon, J. G. Andrews, and F. K. Jondral, "Dualbranch MRC receivers in the cellular downlink under spatial interference correlation," in Proc. 20th Eur. Wireless Conf., May 2014, pp. 13-18.

[17] J. F. C. Kingman, Poisson Processes. Oxford University Press, 1993 